\title{
The Influence of Specimen Size and Distance to a Surface on Resistive Moisture Content Measurements in Wood
}

\author{
Maria Fredriksson, ${ }^{1}$ Johan Claesson, ${ }^{2}$ and Lars Wadsö ${ }^{1}$ \\ ${ }^{1}$ Division of Building Materials, Lund University, Box 118, 22100 Lund, Sweden \\ ${ }^{2}$ Division of Building Physics, Lund University, Box 118, 22100 Lund, Sweden \\ Correspondence should be addressed to Maria Fredriksson; maria.fredriksson@byggtek.lth.se
}

Received 14 November 2014; Revised 3 February 2015; Accepted 3 February 2015

Academic Editor: Carla Roque

Copyright ( 2015 Maria Fredriksson et al. This is an open access article distributed under the Creative Commons Attribution License, which permits unrestricted use, distribution, and reproduction in any medium, provided the original work is properly cited.

\begin{abstract}
The moisture content of wood is commonly determined by measuring the electrical resistance between two electrodes inserted in the wood. However, problems using this method close to wood surfaces were reported in a previous study. In the present study, the effect of the distance to a surface and the specimen size on the measured electrical resistance was studied analytically as follows. The two electrodes create an electrical potential in the wood specimen. The boundary condition for the electrical potential is that the electrical current across all specimen surfaces is zero, which is achieved by using a suitable array of mirror sources. The analytical solution for the electrical potential was used to analyse the influence of the distance from the electrodes to the specimen surface as well as the size of the specimen. In addition, the error in moisture content was evaluated. The effect of the distance to a surface and the specimen size depended on the equivalent radius of the electrodes; if large electrodes are used in small specimens or close to surfaces, there is a risk that a higher resistance is measured which results in slightly lower measured moisture content than the actual moisture content of the specimen.
\end{abstract}

\section{Introduction}

Wood is a hygroscopic material and the moisture content of the wood thus changes with the relative humidity of the surrounding air. The moisture content affects wood properties such as strength and dimensional stability and, in addition, biological degradation is also closely related to the moisture content (see, e.g., [1]). The moisture content is defined as mass of water divided by the mass of the dry wood and is usually determined by the gravimetric method, that is, by mass determination of both the wet piece of wood and after drying at $103^{\circ} \mathrm{C}$. Another common method for moisture content determination is to use a moisture content meter. This method is less accurate than the gravimetric method but is convenient since it enables in situ measurements in wood structures and gives an instant value of the moisture content. The most common moisture content meters determine the moisture content by measuring the electrical resistance or electrical conductance between two electrodes that are inserted in the wood. Since the electrical resistance decreases when the moisture content increases (see, e.g., [2]), the moisture content can be calculated from the measured electrical resistance if the relationship between the resistance and the moisture content is known. The design of the electrodes varies, but usually pin-type electrodes with tapered ends are used (see, e.g., [3]), but there are also examples of other electrode designs (e.g., [4-7]). The electrodes are often insulated so that the electrical conductance is measured between the tips of the electrodes. Apart from resistance-type moisture content meters, there are also capacitance moisture content meters that measure the dielectric constant and power-loss type moisture contents meters that measure the dielectric loss factor, but these types are much less common than the resistance-type moisture content meters [1].

Various factors, both wood properties and experimental variables, can affect the electrical conductance of wood and thus moisture content measurements. Examples of such factors are temperature, wood species, structural orientation, density, chemical constituents, and extractives [8]. Among these factors, the temperature affects the electrical resistance the most [9] and temperature correction is therefore 
necessary. The electrical resistance is also affected by wood species to a large extent since different species contain different amount of extractives and have different densities and different lignin content [8]. The relationship between electrical resistance and moisture content has therefore been determined for different wood species, see, for example, [3]. Preservative treatment of wood with salts generally decreases the electrical resistance and the use of a moisture content meter thus results in a moisture content reading that is higher than the actual moisture content [3]. Moisture content meters are therefore generally not used for preservative treated wood since it is not possible to correct for these errors [3]. Due to polarization effects, the measured electrical resistance is affected by the time during which the voltage is applied [9]. Therefore, the polarity is switched when measuring the electrical conductivity for moisture content determination; see, for example, [7]. The measurements in the verification experiments in the present study were made by applying a voltage of $2 \mathrm{~V}$ during $0.5 \mathrm{~s}$ after which a first reading was made during $0.5 \mathrm{~s}$. The polarity was then switched and after $0.5 \mathrm{~s}$ a second reading was made during $0.5 \mathrm{~s}$. This is similar to how most wood moisture meters work. It is thus a simple measurement with the only aim of acquiring a resistance value that can be related to the moisture content of the wood. These types of devices work with voltage steps which contain both high and low frequency components, but as each step is rather long $(0.5 \mathrm{~s})$ a measurement can be considered to be quasi-stationary (DC electrical resistance moisture measurement).

Skaar [8] claimed that the size of the sample is of little importance since the resistance is concentrated on the vicinity of the electrodes. However, Nore and Thue [10] reported problems using resistive moisture content measurements close to a surface. They found that the moisture content measured by the resistive method was lower $3 \mathrm{~mm}$ below the surface than in the middle of a $23 \mathrm{~mm}$ thick board even though the board should have a uniform moisture content. Their results thus indicate that the measured moisture content is affected if measurements are made close to a surface.

This paper presents an analytical study on the influence of the distance to a surface and specimen size on the measured electrical resistance between two electrodes inserted in a specimen. The error in resistance was also translated to error in moisture content by using a relationship between electrical resistance and moisture content from the literature.

\section{Theory}

The electrical current $I$ obeys Ohm's law:

$$
I=\frac{V}{R}
$$

where $V$ is the difference in electrical potential and $R$ is the electrical resistance. The electrical resistance is the inverse of the electrical conductance $G$ :

$$
R=\frac{1}{G}
$$

The electrical potential $V$ from a point source at a distance $r$ from the source is

$$
V=\frac{I}{4 \pi \sigma} \cdot \frac{1}{r}
$$

where $\sigma$ is the electrical conductivity which is the inverse of the electrical resistivity $\rho$ :

$$
\sigma=\frac{1}{\rho} .
$$

The distance $r$ is

$$
r=\sqrt{\left(x-x_{1}\right)^{2}+\left(y-y_{1}\right)^{2}+\left(z-z_{1}\right)^{2}},
$$

where $\left(x_{1}, y_{1}, z_{1}\right)$ are the Cartesian coordinates of the point source.

In the following sections, we will derive equations for the electrical potential in limited size specimens with two electrodes. We will first do this for the one-dimensional case and then extend this solution to the three-dimensional case. It should be noted that the potential differences between the two electrodes are dependent on the size of the electrodes, which we assume to be spherical with a certain equivalent radius.

2.1. Two Electrodes on the $x$-Axis. The electrical potential from the positive and negative electrodes at $\left(x_{1}, y_{1}, z_{1}\right)$ and $\left(x_{2}, y_{2}, z_{2}\right)$ is

$$
\begin{aligned}
& V(x, y, z) \\
&=\frac{I}{4 \pi \sigma}\left(\frac{1}{\sqrt{\left(x-x_{1}\right)^{2}+\left(y-y_{1}\right)^{2}+\left(z-z_{1}\right)^{2}}}\right. \\
&\left.-\frac{1}{\sqrt{\left(x-x_{2}\right)^{2}+\left(y-y_{2}\right)^{2}+\left(z-z_{2}\right)^{2}}}\right) .
\end{aligned}
$$

The rectangular cuboidal wood sample occupies the region $0<x<L_{x}, 0<y<L_{y}, 0<z<L_{z}$. The electrical current in any point is equal to the gradient of the electrical potential times the electrical conductivity $\sigma$. The boundary conditions at the six wood surfaces are that the electrical current perpendicular to the surfaces is zero. The boundary condition at the surfaces $x=0$ and $x=L_{x}$ is

$$
\begin{aligned}
& \frac{\partial V}{\partial x}=0, \quad x=0, x=L_{x}, \\
& 0<y<L_{y}, \quad 0<z<L_{z} .
\end{aligned}
$$

There are corresponding conditions in the $y$ and $z$ directions. These conditions are achieved by putting suitable arrays of mirror sources outside the wood sample as described below.

Figure 1(a) shows the electrodes placed at $\left(x_{1}, 0,0\right)$ and $\left(x_{2}, 0,0\right)$. To obtain zero gradient at $x=0$, two mirror sources are placed at $\left(-x_{1}, 0,0\right)$ and $\left(-x_{2}, 0,0\right)$ (Figure $\left.1(b)\right)$. 


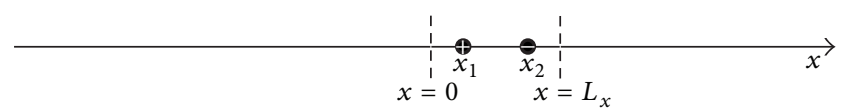

(a)

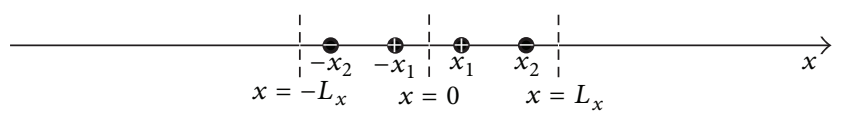

(b)

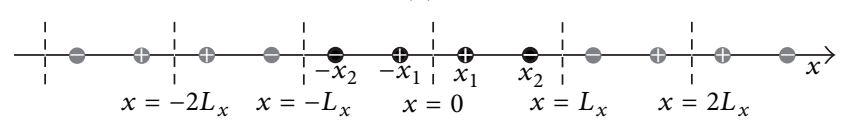

(c)

Figure 1: (a) Two electrodes in a specimen with length $L_{x}$. (b) The electrodes and two mirror sources (the unit cell). (c) The unit cell is repeated along the $x$-axis.

This element from $-L_{x}$ to $L_{x}$ is symmetric around zero and is considered a unit cell. The gradient $\partial V / \partial x$ at $x=0$ is zero due to symmetry. To achieve zero gradient at $x=L_{x}$, this unit cell is repeated to both the left and the right (Figure 1(c)). If more unit cells are placed next to each other in both directions, the gradient at $x=L_{x}$ converges towards zero. The gradient at $x=L_{x}$ from all sources but the last two at the far left is zero due to symmetry. The gradient from the last two plus and minus sources tends to zero as $N$ increases. The coordinates of the positive point source and its mirror sources are

$$
\begin{array}{r}
x=\left(1-2 m_{x}\right) x_{1}+n_{x} \cdot 2 L_{x} \\
m_{x}=0,1 \quad n_{x}=0, \pm 1, \ldots, \pm N,
\end{array}
$$

and the coordinates for the negative point source and its mirror sources are

$$
\begin{array}{r}
x=\left(1-2 m_{x}\right) x_{2}+n_{x} \cdot 2 L_{x} \\
m_{x}=0,1 \quad n_{x}=0, \pm 1, \ldots, \pm N,
\end{array}
$$

where $N$ is the number of repetitions of the unit cell to the left and right, respectively.

Equation (3) gives the electrical potential of a point source at a distance $r$ from the source. Since this study is limited to comparing ratios between electrical potentials when the electrodes are placed in specimens with different size or in different points in the same specimen, $I / 4 \pi \sigma$ in (3) is set to 1 henceforth.

The potential for the positive point source and its mirror source in the unit cell is

$$
V_{1}(x, y, z)=\sum_{m_{x}=0}^{1} \frac{1}{\sqrt{\left(x-x_{1}+m_{x} 2 x_{1}\right)^{2}+y^{2}+z^{2}}}
$$

and the potential from the negative point source and its mirror source in the unit cell is

$$
V_{2}(x, y, z)=\sum_{m_{x}=0}^{1} \frac{1}{\sqrt{\left(x-x_{2}+m_{x} 2 x_{2}\right)^{2}+y^{2}+z^{2}}} .
$$

The total potential from both point sources and their mirror sources in the unit cell is

$$
V_{12}(x, y, z)=V_{1}(x, y, z)-V_{2}(x, y, z) .
$$

If the unit cell is repeated $N$ times as shown in Figure 1(c), the total potential from the point sources and all their mirror sources is

$$
V(x, y, z, N)=\sum_{n_{x}=-N}^{N} V_{12}\left(x-n_{x} 2 L_{x}, y, z\right) .
$$

\subsection{Two Electrodes in a Rectangular Cuboidal Specimen}

2.2.1. Coordinates. The above approach is used also in the three-dimensional case. The specimen is then in the region $0<x<L_{x}, 0<y<L_{y}, 0<z<L_{z}$. The coordinates of the positive point source and its mirror sources when the unit cell is repeated $N$ times along the $x$-, $y$-, and $z$-axis are

$$
\begin{aligned}
& x=\left(1-2 m_{x}\right) x_{1}+n_{x} 2 L_{x}, \quad m_{x}=0,1, n_{x}=0, \pm 1, \ldots, \pm N, \\
& y=\left(1-2 m_{y}\right) y_{1}+n_{y} 2 L_{y}, \quad m_{y}=0,1, n_{y}=0, \pm 1, \ldots, \pm N, \\
& z=\left(1-2 m_{z}\right) z_{1}+n_{z} 2 L_{z}, \quad m_{z}=0,1, n_{z}=0, \pm 1, \ldots, \pm N
\end{aligned}
$$

and the coordinates of the negative point source and its mirror sources are

$$
\begin{aligned}
& x=\left(1-2 m_{x}\right) x_{2}+n_{x} 2 L_{x}, \quad m_{x}=0,1, n_{x}=0, \pm 1, \ldots, \pm N, \\
& y=\left(1-2 m_{y}\right) y_{2}+n_{y} 2 L_{y}, \quad m_{y}=0,1, n_{y}=0, \pm 1, \ldots, \pm N, \\
& z=\left(1-2 m_{z}\right) z_{2}+n_{z} 2 L_{z}, \quad m_{z}=0,1 n_{z}=0, \pm 1, \ldots, \pm N .
\end{aligned}
$$

2.2.2. Electric Potential. The potential from the positive point sources $\left(V_{1}\right)$ in the unit cell is

$$
\begin{aligned}
V_{1}=\sum_{m_{x}=0}^{1} \sum_{m_{y}=0}^{1} \sum_{m_{z}=0}^{1} & \left(\left(x-\left(1-2 m_{x}\right) x_{1}\right)^{2}\right. \\
& +\left(y-\left(1-2 m_{y}\right) y_{1}\right)^{2} \\
& \left.+\left(z-\left(1-2 m_{z}\right) z_{1}\right)^{2}\right)^{-1 / 2}
\end{aligned}
$$

and the potential from the negative point source $\left(V_{2}\right)$ is

$$
\begin{aligned}
V_{2}=\sum_{m_{x}=0}^{1} \sum_{m_{y}=0}^{1} \sum_{m_{z}=0}^{1}( & \left(x-\left(1-2 m_{x}\right) x_{2}\right)^{2} \\
+ & \left(y-\left(1-2 m_{y}\right) y_{2}\right)^{2} \\
& \left.+\left(z-\left(1-2 m_{z}\right) z_{2}\right)^{2}\right)^{-1 / 2} .
\end{aligned}
$$

The total potential from both point sources $\left(V_{12}\right)$ and their mirror sources in the unit cell is

$$
V_{12}(x, y, z)=V_{1}(x, y, z)-V_{2}(x, y, z) .
$$


As in the one-dimensional case, the unit cell is repeated in the three directions. The total potential from the point sources and all their mirror sources when the unit cell is repeated $N$ times along the $x, y$, and $z$-axis is

$$
\begin{aligned}
& V(x, y, z, N) \\
& =\sum_{n_{x}=-N}^{N} \sum_{n_{y}=-N}^{N} \sum_{n_{z}=-N}^{N} V_{12}\left(x-n_{x} 2 L_{x}, y-n_{y} 2 L_{y}, z-n_{z} 2 L_{z}\right) .
\end{aligned}
$$

2.2.3. Difference in Potential between Two Electrodes. The difference in potential between two electrodes $(\Delta V)$ for the $3 \mathrm{D}$ case is calculated as

$$
\Delta V=V_{12}(x, y, z)-V_{12}(x, y, z) .
$$

In reality, the electrodes are not point sources and the potential difference between two electrodes will depend on the shape and the size of the electrodes. We have chosen to work with spherical electrodes and numerically evaluated (18) in six points on these spherical surfaces. The potential difference was calculated as the mean value of the potential in six points at the sphere surface:

$$
\begin{array}{ll}
P_{1+}:\left(x_{1}+r_{e}, y_{1}, z_{1}\right), & P_{1-}:\left(x_{2}+r_{e}, y_{2}, z_{2}\right), \\
P_{2+}:\left(x_{1}-r_{e}, y_{1}, z_{1}\right), & P_{2-}:\left(x_{2}-r_{e}, y_{2}, z_{2}\right), \\
P_{3+}:\left(x_{1}, y_{1}+r_{e}, z_{1}\right), & P_{3-}:\left(x_{2}, y_{2}+r_{e}, z_{2}\right), \\
P_{4+}:\left(x_{1}, y_{1}-r_{e}, z_{1}\right), & P_{4-}:\left(x_{2}, y_{2}-r_{e}, z_{2}\right), \\
P_{5+}:\left(x_{1}, y_{1}, z_{1}+r_{e}\right), & P_{5-}:\left(x_{2}, y_{2}, z_{2}+r_{e}\right), \\
P_{6+}:\left(x_{1}, y_{1}, z_{1}-r_{e}\right), & P_{6-}:\left(x_{2}, y_{2}, z_{2}-r_{e}\right),
\end{array}
$$

where $r_{e}$ is the equivalent radius of the electrodes. The equivalent radius is the radius of the sphere if the electrodes are spherical. However, electrodes are generally not spherical and there is also generally a surface resistance between the electrodes and the wood. Which equivalent radius that corresponds to the electrodes used therefore has to be determined experimentally for different electrode types. This means that $r_{e}$ is to be evaluated from measured data which are matched to calculated potential differences.

2.3. Size of $N$. In order to determine which size of $N$ is needed, that is, how many times the unit cell needs to be repeated, the difference in potential was calculated for different $N$. The calculations were performed for electrodes in the middle of a specimen with $L_{x}=L_{y}=L_{z}=100 \mathrm{~mm}$ as well as for a smaller specimen with $L_{x}=L_{y}=L_{z}=11 \mathrm{~mm}$. The distance between the electrodes was $10 \mathrm{~mm}$, and $r_{e}$ was set to $0.5 \mathrm{~mm}$. The results are shown in Figure 2 and the different values of $N$ only gave small differences in $\Delta V / \Delta V_{\infty}$ and a value of $N$ that is equal to or larger than 5 is therefore enough. The effect of the size of $N$ was larger for the small specimen.

\section{Influence of Various Parameters on the Measured Conductance}

3.1. Reference Case: Two Electrodes in an Infinite Volume. To determine how much the measured conductance is affected by the extent of the specimen, the potential between two electrodes in an infinite volume was calculated and used as a reference case. The electric potential for the positive $\left(V_{1 \infty}\right)$ and negative point source $\left(V_{2 \infty}\right)$, respectively, is

$$
\begin{aligned}
& V_{1 \infty}(x, y, z)=\frac{1}{\sqrt{\left(x-x_{1}\right)^{2}+\left(y-y_{1}\right)^{2}+\left(z-z_{1}\right)^{2}}}, \\
& V_{2 \infty}(x, y, z)=\frac{1}{\sqrt{\left(x-x_{2}\right)^{2}+\left(y-y_{2}\right)^{2}+\left(z-z_{2}\right)^{2}}} .
\end{aligned}
$$

The total potential from both electrodes is

$$
V_{12 \infty}(x, y, z)=V_{1 \infty}(x, y, z)-V_{2 \infty}(x, y, z) .
$$

The difference in potential of the two electrodes is then calculated as the mean difference at different points at the sphere surface:

$$
\begin{gathered}
\Delta V_{\infty 1}=V_{12 \infty}\left(x_{1}+r_{e}, y_{1}, z_{1}\right)-V_{12 \infty}\left(x_{2}+r_{e}, y_{2}, z_{2}\right), \\
\Delta V_{\infty 2}=V_{12 \infty}\left(x_{1}-r_{e}, y_{1}, z_{1}\right)-V_{12 \infty}\left(x_{2}-r_{e}, y_{2}, z_{2}\right), \\
\Delta V_{\infty 3}=V_{12 \infty}\left(x_{1}, y_{1}+r_{e}, z_{1}\right)-V_{12 \infty}\left(x_{2}, y_{2}+r_{e}, z_{2}\right), \\
\Delta V_{\infty 4}=V_{12 \infty}\left(x_{1}, y_{1}-r_{e}, z_{1}\right)-V_{12 \infty}\left(x_{2}, y_{2}-r_{e}, z_{2}\right), \\
\Delta V_{\infty 5}=V_{12 \infty}\left(x_{1}, y_{1}, z_{1}+r_{e}\right)-V_{12 \infty}\left(x_{2}, y_{2}, z_{2}+r_{e}\right), \\
\Delta V_{\infty 6}=V_{12 \infty}\left(x_{1}, y_{1}, z_{1}-r_{e}\right)-V_{12 \infty}\left(x_{2}, y_{2}, z_{2}-r_{e}\right), \\
\Delta V_{\infty \text { mean }} \\
\quad=\frac{\Delta V_{\infty 1}+\Delta V_{\infty 2}+\Delta V_{\infty 3}+\Delta V_{\infty 4}+\Delta V_{\infty 5}+\Delta V_{\infty 66}}{6} .
\end{gathered}
$$

3.2. The Influence of the Distance to a Surface. The influence of the distance to a surface on the measured resistance was investigated by placing the electrodes in a specimen with a large volume and varying the position of the electrodes along the $z$-axis towards one of the surfaces. The specimen size was set to $100 \times 100 \times 100 \mathrm{~m}^{3}$, that is, $L_{x}=L_{y}=L_{z}=$ $100 \mathrm{~m}$, since this large specimen size would ensure that the calculated difference in potential would not be disturbed by volume effects (see Section 3.3). The difference in potential $(\Delta V)$ between the two electrodes was then determined for different $r_{e}$ and $N=5$ as described in Section 2.2.3 for the different positions at the $z$-axis. The minimum distance to the surface was set equal to $r_{e}$ and the spacing between the electrodes was $10 \mathrm{~mm}$. The results in relation to the potential in an infinite volume (see Section 4.1) are shown in Figure 3.

3.3. The Influence of the Specimen Volume. The influence of specimen volume was studied by calculating the difference in 


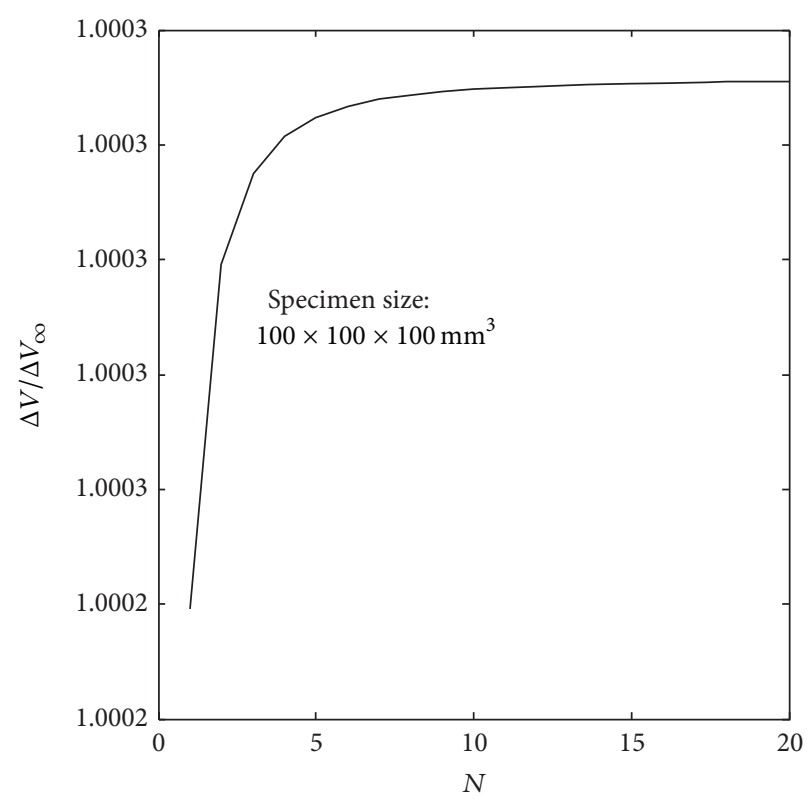

(a)

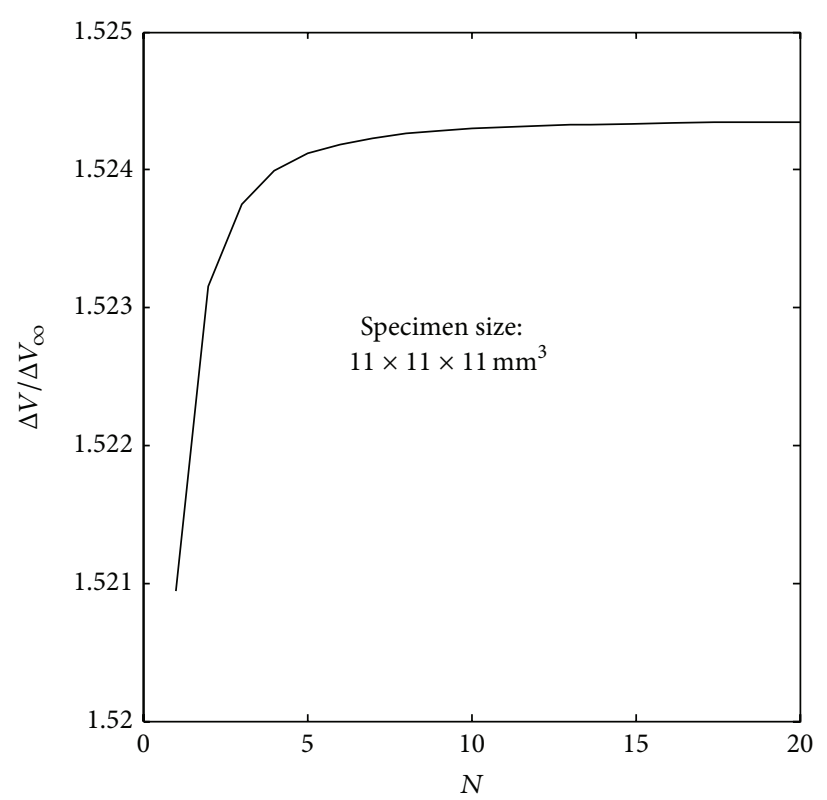

(b)

Figure 2: The ratio $\Delta V / \Delta V_{\infty}$ as a function of $N$ for a cubic specimen with side length $100 \mathrm{~mm}$ (a) and smaller cubic specimen with side length $11 \mathrm{~mm}$ (b).

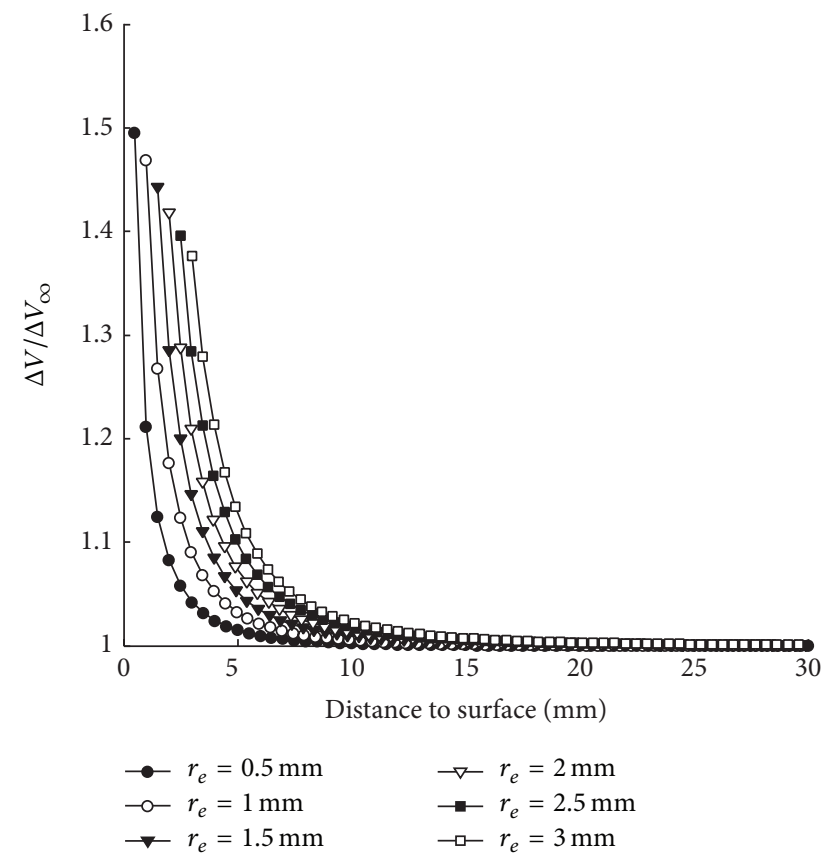

FIgURE 3: The difference in potential calculated in a specimen at different distances to a surface $(\Delta V)$ in relation to the difference in potential in an infinite volume $\left(\Delta V_{\infty}\right)$. The calculations were performed for several equivalent radii $\left(r_{e}\right)$.

potential (see Section 2.2.3) between two electrodes inserted in the middle of a cubic specimen. The distance between the electrodes was $10 \mathrm{~mm}$. The sides of the cubic specimen $\left(L_{x}\right.$, $L_{y}$, and $L_{z}$ ) were set to values between $2 \cdot r_{e}+10 \mathrm{~mm}$ and
$80 \mathrm{~mm}$. The difference in potential for the different specimen volumes was determined for different $r_{e}$ and $N$ was set to 5. The calculated difference in potential was divided by the potential between two electrodes in an infinite volume (see Section 4.1) and the results are shown in Figure 4. The results show that cubic specimens with a side exceeding about $70 \mathrm{~mm}$ give a similar potential difference as if the measurements were made in an infinite volume. The results for smaller specimens depend on the size and the equivalent radius.

3.4. Error in Moisture Content. Depending on specimen size, distance to surface, and equivalent radius, the ratio $\Delta V / \Delta V_{\infty}$ was between 1 and 1.6 for the studied cases (see Figures 3 and 4). In order to determine how much this error in resistance affects the moisture content, additional calculations were performed. The function describing the relationship between the moisture content and electrical resistance given by Fredriksson et al. [7] was used. Resistances corresponding to moisture contents $(\mathrm{kg} / \mathrm{kg})$ of $0.15,0.20,0.25$, and 0.3 were multiplied by ratios $(r)$ between 1 and 1.6. The error in moisture content was then determined by

$$
\Delta u=u(R)-u(r \cdot R) .
$$

The results are shown in Figure 5.

As an example, calculations for two specimens used by Fredriksson et al. [7] were performed. Here, two types of small specimens were used: circular discs with diameter $60 \mathrm{~mm}$ and thickness $10 \mathrm{~mm}$ and rectangular specimens with dimensions $30 \times 20 \times 10 \mathrm{~mm}^{3}$. The difference in potential between the electrodes for both specimen types was calculated as described in Section 2.2.3 and the circular specimens 


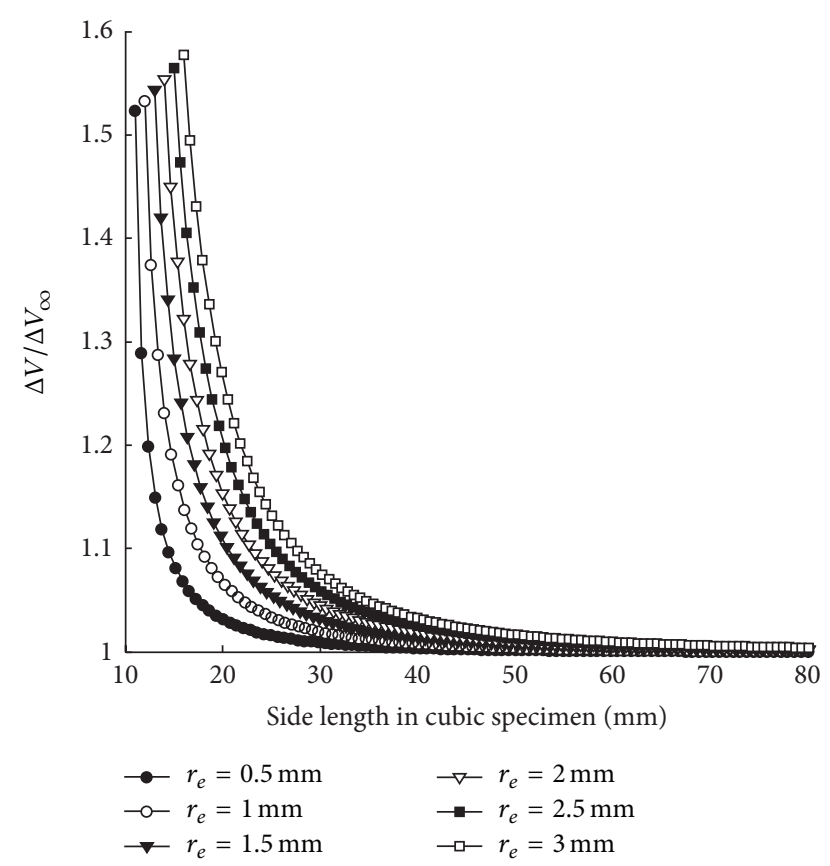

FIgURE 4: The difference in potential $(\Delta V)$ calculated in cubic specimens with different volumes in relation to the difference in potential in an infinite volume $\left(\Delta V_{\infty}\right)$. The calculations were performed for several equivalent radii $\left(r_{e}\right)$.

were modelled as rectangular specimens with side $60 \mathrm{~mm}$ and thickness $10 \mathrm{~mm}$. The distance between the electrodes was $10 \mathrm{~mm}$. The ratio $\Delta V / \Delta V_{\infty}$ was then calculated. For $r_{e}=0.8 \mathrm{~mm}$, which was the actual radius of the electrodes, the ratio $\Delta V / \Delta V_{\infty}$ was 1.07 for the $60 \times 60 \times 10 \mathrm{~mm}^{3}$ specimen and 1.13 for the $30 \times 20 \times 10 \mathrm{~mm}^{3}$ specimen. This means that the measured resistance is $7 \%$ and $13 \%$ higher than that if the measurement was made in an infinite volume. According to Figure 5, this gives an absolute error in moisture content of about $0.002 \mathrm{~kg} / \mathrm{kg}$ and $0.004 \mathrm{~kg} / \mathrm{kg}$ at $20 \%$ moisture content for the two specimen sizes, respectively, and at $30 \%$ moisture content the error for the same specimens increases to about $0.005 \mathrm{~kg} / \mathrm{kg}$ and $0.008 \mathrm{~kg} / \mathrm{kg}$. However, the equivalent radius is most likely higher than the actual radius of the electrodes since it includes the surface resistance betweeen the electrodes and the wood. A larger equivalent radius means that the ratio $\Delta V / \Delta V_{\infty}$ increases as well as the error in moisture content. For example, for $r_{e}=2.5 \mathrm{~mm}$, the ratio $\Delta V / \Delta V_{\infty}$ increases to 1.29 for the $60 \times 60 \times 10 \mathrm{~mm}^{3}$ specimen and 1.51 for the $30 \times 20 \times 10 \mathrm{~mm}^{3}$ specimen which according to Figure 5 give absolute errors in moisture content of $0.007 \mathrm{~kg} / \mathrm{kg}$ and $0.012 \mathrm{~kg} / \mathrm{kg}$, respectively.

\section{Verification}

4.1. Experimental Procedure. In order to verify the model described in the previous sections, an experiment was set up. Electrodes as described by Fredriksson et al. [7] were inserted in the middle of a specimen with dimensions $22 \times$ $95 \times 270 \mathrm{~mm}^{3}$ (radially $\times$ tangentially $\times$ longitudinally) at

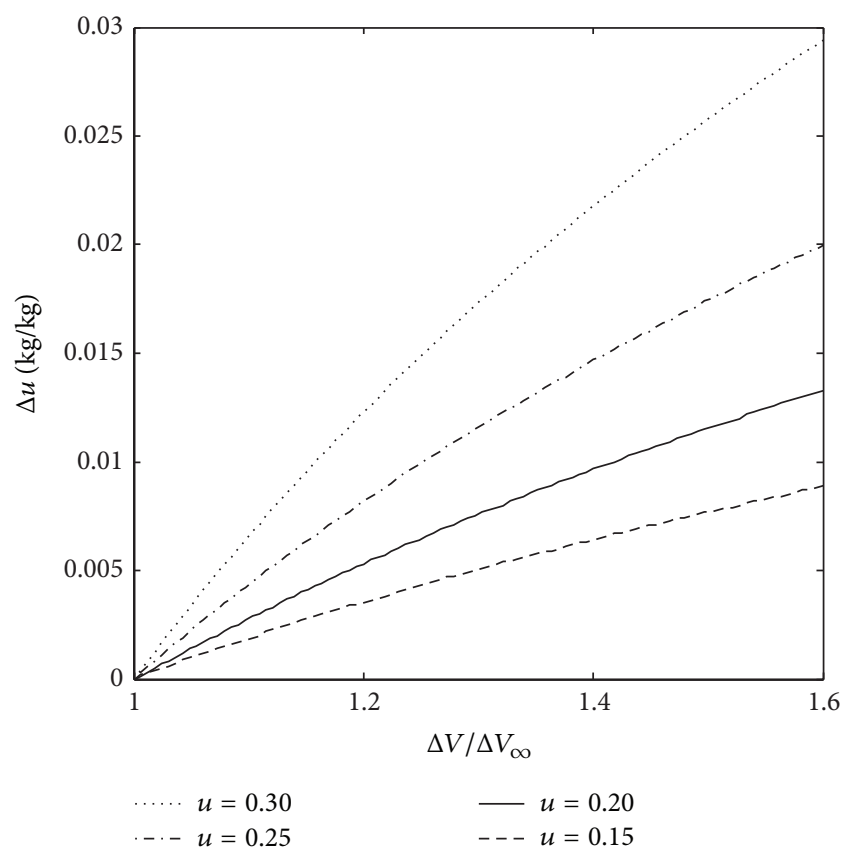

FIGURE 5: The absolute error in moisture content $(\Delta u)$ for different ratios $\Delta V / \Delta V_{\infty}$ determined for four moisture content levels $(u)$.

three depths: $3 \mathrm{~mm}$ below the surface, $11 \mathrm{~mm}$ below the surface, and $19 \mathrm{~mm}$ below the upper surface, that is, $3 \mathrm{~mm}$ above the lower surface. The specimen with the electrodes was dried at $30^{\circ} \mathrm{C}$ (approximately $30 \%$ relative humidity) in order to avoid moisture gradients and ensure that the whole specimen was later on the absorption isotherm. After drying, the specimen was placed at $20^{\circ} \mathrm{C}$ in a box with $75.5 \%$ relative humidity which was generated by a saturated salt solution $(\mathrm{NaCl})$. The electrical conductance in the different points of measurements was logged during four months after which equilibrium was reached.

4.2. Evaluation. The conductance at equilibrium at the different depths is shown in Figure 6. Each value in the figure is the average of the last 10 logged values. The median electrical conductance is also shown. The corresponding electrical resistance to the median values at each depth was calculated by (2) and the relative difference in resistance at the different depths was determined. The median resistance in the points $3 \mathrm{~mm}$ from the upper and the lower surfaces was about $30 \%$ higher than the median resistance at $11 \mathrm{~mm}$ depth. From Figure 5, it is seen that an increase in resistance of $30 \%$ gives an absolute error in moisture content of about $0.005 \mathrm{~kg} / \mathrm{kg}$, that is, $0.5 \%$ moisture content. This is similar to the error that was seen by Nore and Thue [10] which suggests that the small distance to the surface can be the cause of the lower moisture contents measured close to the surface in the boards in their study.

As the influence of the distance to a surface depends on the electrode radius, the equivalent radius that corresponds to the results in Figure 6 was calculated. Using the model 

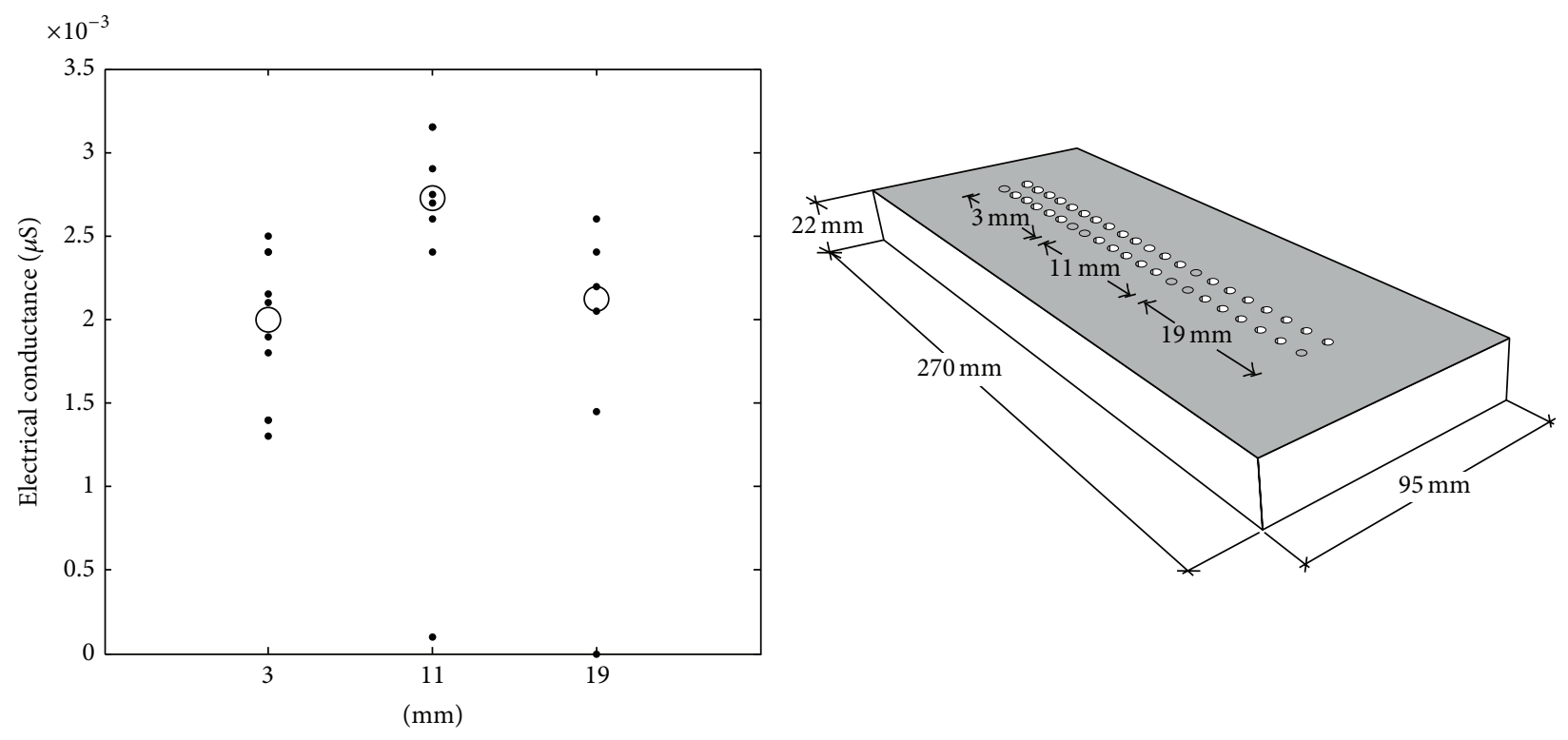

O Median

Figure 6: The electrical conductance measured at three depths in the points indicated by the illustration to the right. The median value at each depth is marked by a circle.

described in Section 2.2, the results indicate that the equivalent radius was about $3 \mathrm{~mm}$. However, due to issues related to the mounting of the electrodes in this particular experiment, the value is uncertain and does not necessarily correspond to the equivalent radius for the electrodes mounted in the specimens described in Section 3.4.

\section{Conclusions}

The size of the specimen and the distance to a surface do affect the measured resistance. The absolute error in moisture content is small but increases with increasing moisture content. Electrodes with a large equivalent radius used in small specimens or close to surfaces may give slightly higher resistance values and thereby lower moisture contents than that if the measurements are made in a larger volume.

\section{Conflict of Interests}

The authors declare that there is no conflict of interests regarding the publication of this paper.

\section{Acknowledgments}

This work is part of WoodBuild, a research programme within the Sectoral Research and Development Programme 2006-2012 for the Swedish forest-based industry. This Programme is jointly funded by the government, industry, and other stakeholders with interests related to the Swedish forest-based industry. Funding from the Swedish Research Council, Formas, is also gratefully acknowledged.

\section{References}

[1] J. M. Dinwoodie, Timber, Its Nature and Behavior, E \& FN Spon, London, UK, 2000.

[2] A. J. Stamm, "The electrical resistance of wood as a measure of its moisture content," Industrial \& Engineering Chemistry, vol. 19, no. 9, pp. 1021-1025, 1927.

[3] W. L. James, "Electric moisture meters for wood," Tech. Rep. FPL-GTR-6, United States Department of Agriculture, Forest Service, Forest Products Laboratory, Madison, Wis, USA, 1988.

[4] H. Derbyshire and E. R. Miller, "Moisture conditions in coated exterior wood Part 2: the relation between coating permeability and timber moisture content," Journal of the Institute of Wood Science, vol. 14, no. 4, pp. 162-168, 1997.

[5] H. Derbyshire and E. R. Miller, "Moisture conditions in coated exterior wood Part 3. Moisture content during natural weathering," Journal of the Institute of Wood Science, vol. 14, no. 4, pp. 169-174, 1997.

[6] C. Brischke, A. O. Rapp, and R. Bayerbach, "Measurement system for long-term recording of wood moisture content with internal conductively glued electrodes," Building and Environment, vol. 43, no. 10, pp. 1566-1574, 2008.

[7] M. Fredriksson, L. Wadsö, and P. Johansson, "Small resistive wood moisture sensors: a method for moisture content determination in wood structures," European Journal of Wood and Wood Products, vol. 71, no. 4, pp. 515-524, 2013.

[8] C. Skaar, Wood-Water Relations, Springer, Berlin, Germany, 1988.

[9] C. Skaar, "Some factors involved in the electrical determination of moisture gradients in wood," Forest Products Journal, vol. 14, no. 6, pp. 239-243, 1964.

[10] K. Nore and J. V. Thue, "Evaluation of moisture pins in wooden claddings," in Proceedings of the 8th Symposium on Building Physics in the Nordic Countries, Copenhagen, Denmark, June 2008. 


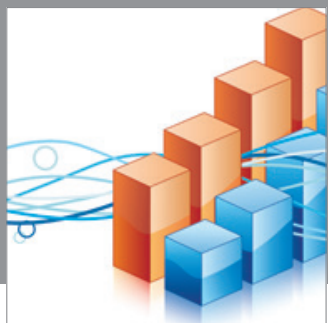

Advances in

Operations Research

mansans

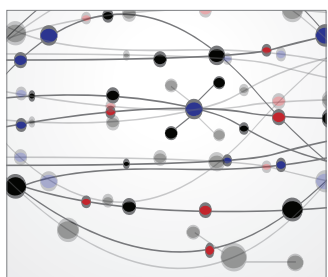

The Scientific World Journal
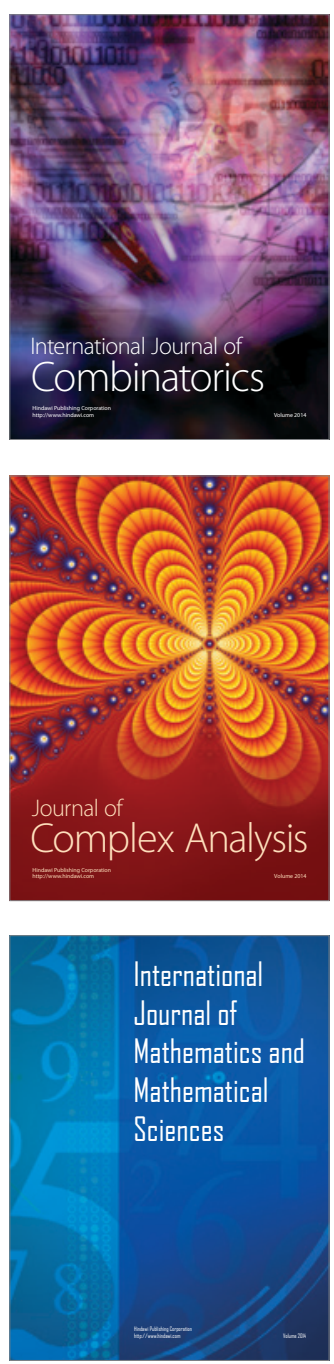
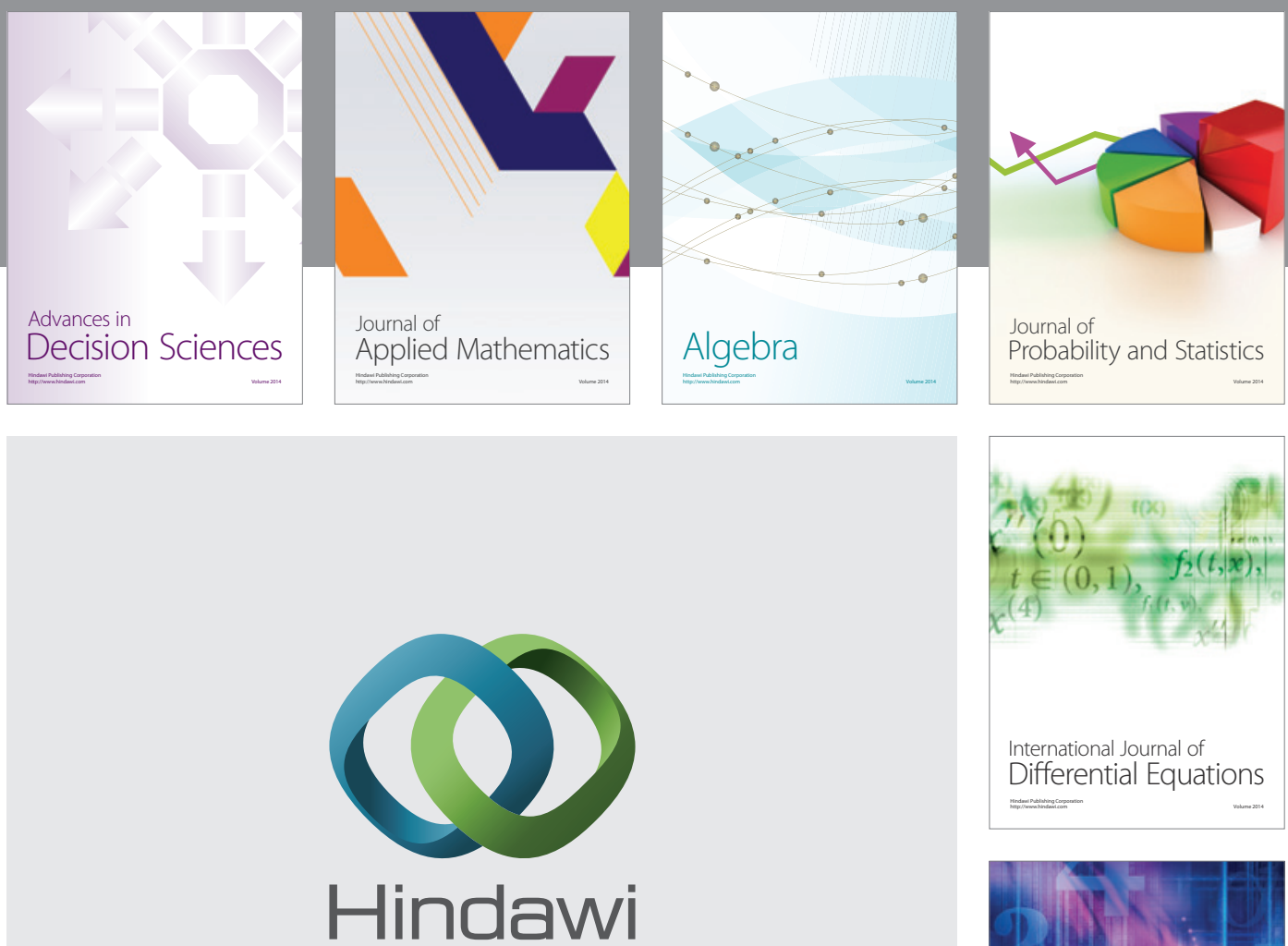

Submit your manuscripts at http://www.hindawi.com
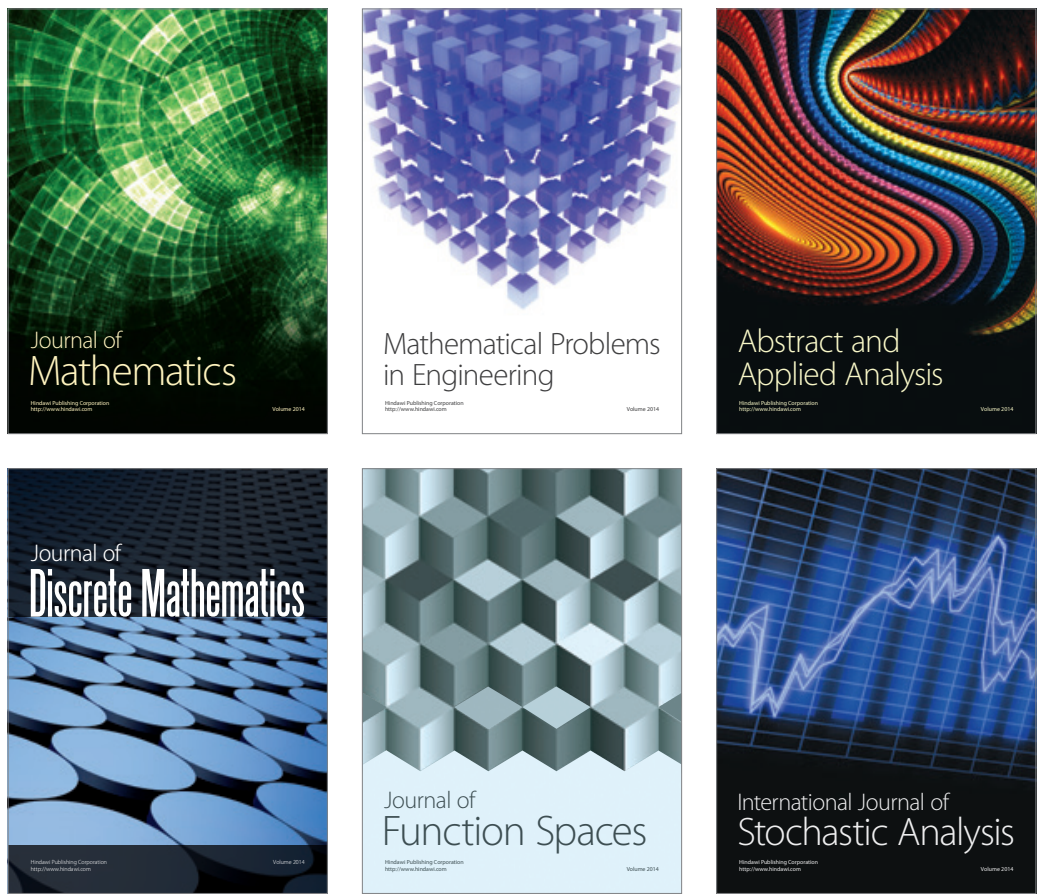

Journal of

Function Spaces

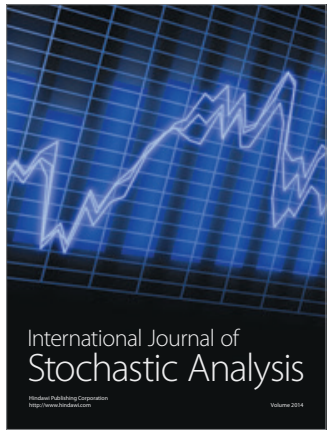

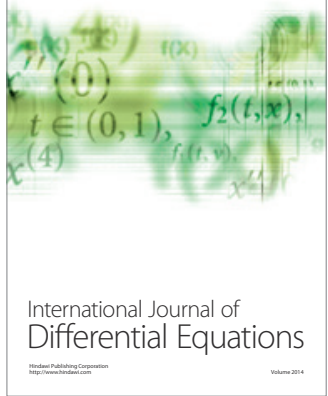
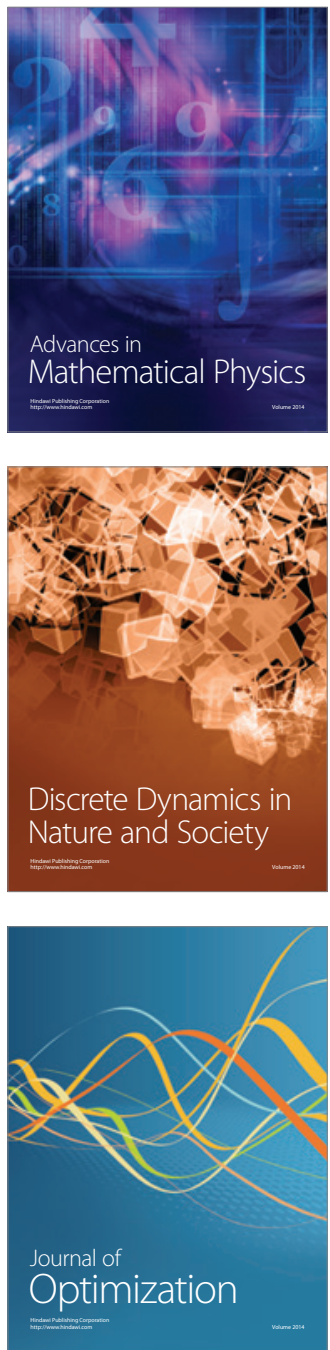